\title{
Linear lichen planus associated with primary aldosteronism
}

\section{Natsuko Matsumura, Toshiyuki Yamamoto}

\author{
Department of Dermatology, Fukushima Medical University, Fukushima, Japan
}

Corresponding author: Natsuko Matsumura, MD, E-mail: natsukom@fmu.ac.jp

Sir,

Lichen planus (LP) is categorized as a chronic inflammatory skin disease of unknown etiology that involves immune reactions. It is characterized by flattopped, polygonal, violaceous papules and plaques. It has various clinical presentations, such as classical LP, hypertrophic LP, LP pigmentosus, and linear LP (LLP). Primary aldosteronism (PA) is known to pose a higher risk of causing multiple autoimmune diseases [1]. Herein, we report a case with LLP and PA present at the same time.

A 72-year-old Japanese female presented herself to our hospital with a three-month history of slightly itchy skin lesions on the lower right leg. A physical examination revealed flat-topped plaques on the lower right limb extending from the middle of the leg to the dorsum of the foot (Fig. la). There was no oral or nail involvement. The patient had a history of hypertension from the age of 62 years and was diagnosed with PA afterward. The patient had been treated with an antihypertensive drug since then without change in internal medication. The patient had no history of a preceding trauma, dental metal fillings, hepatitis, metastatic cancer, or any other infections. A histopathological examination of a skin biopsy from a lesion on the right leg revealed hyperkeratosis, a saw-tooth appearance of the epidermis, and severe liquefaction degeneration. A band-like lymphocytic infiltration was present in the upper dermis (Figs. 2a and 2b), as well as lichenoid infiltration into the dermis composed of CD4+ and CD8+ T lymphocytes. Predominantly, CD8 + T lymphocytes infiltrated into the epidermis (Figs. 2c and 2d). Clinical and histological findings confirmed the diagnosis of LPP.
The patient was treated with a topical corticosteroid. The lesions improved after six months (Fig. lc).

LP is a chronic inflammatory autoimmune disease affecting not only the skin but also the oral and genital mucosas, scalp, and nails. It usually appears as itchy shiny flat-topped papules that may persist for several weeks. It has various clinical presentations, such as classical LP, hypertrophic LP, LP pigmentosus, and linear LP (LLP). LLP is observed in less than $0.2 \%$ of all LP patients [2]. It sometimes occurs alongside autoimmune disorders, viral infections, allergic reactions from certain metals and chemicals, stress, radiation, and genetic conditions [3-5]. Mild cases of lichen planus clear up in weeks to months.

Our case had no history of recent vaccination or episodes of allergic reaction to metals. The patient had been taking eplerenone, an oral aldosterone antagonist, for eight years since the diagnosis of primary aldosteronism, but the medication remained unchanged after blood pressure became well controlled. The lesions improved without change in drugs. We excluded drug-induced LP.

The exact etiology of our patient's LLP is unknown and we speculated that the coexistence of primary aldosteronism could have been one trigger. A study by Herrada et al. showed that aldosterone augmented the activation of CD8 + T cells in a dendritic cell-dependent fashion, and that this condition contributed to inflammatory damage, leading to hypertension and cardiovascular diseases. In addition, stimulation with aldosterone imposed a Thl7 phenotype on CD4+ T cells, which has recently been associated with the promotion of inflammatory and autoimmune diseases [6]. In our case, bilateral adrenal venous sampling revealed high levels of plasma 


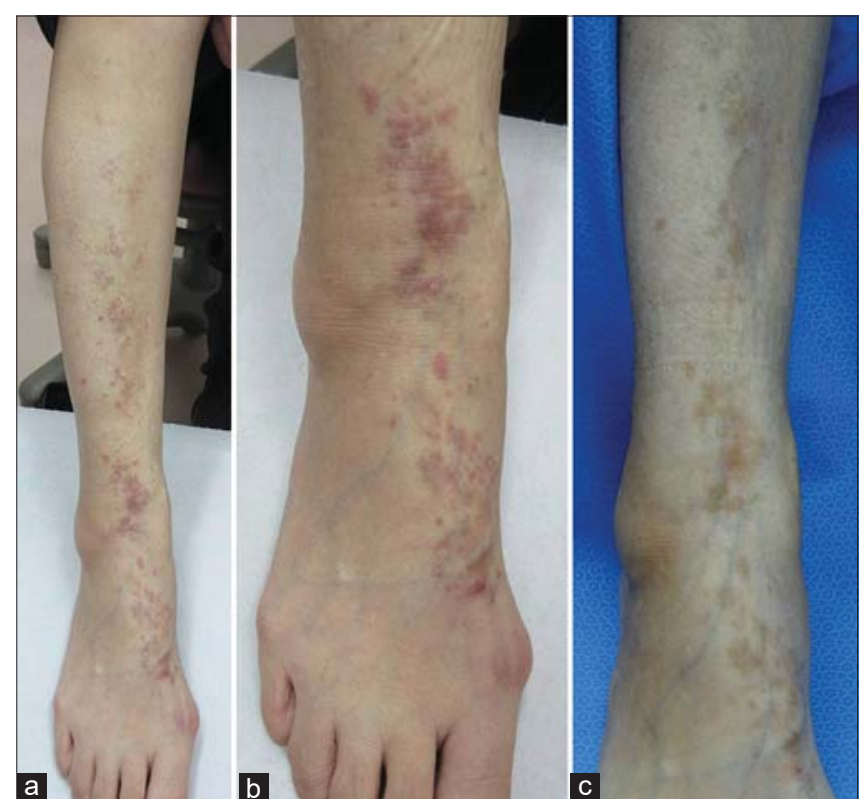

Figure 1: (a-c) Flat-topped plaques on the lower right limb extending from the middle of the leg to the dorsum of the foot.

aldosterone. We presume that the excessive production of aldosterone might have led to an inflammatory state, which may have been promoted by $\mathrm{T}$ cell immunity and led to the abnormal keratinocyte cloning on the patient's right foot to unmasked LLP. We were unable to find any report in the literature of the coexistence of LLP and primary aldosteronism as seen in our case. Recent studies have suggested that lichen planus is associated with an increased risk of cardiovascular comorbidities. Baykal Selcuk et al. observed a positive correlation between the duration of the disease and arterial stiffness in a patient with lichen planus [7]. Our case seems to have needed immediate treatment and careful follow-up.

In summary, primary aldosteronism poses a risk of promoting an inflammatory state and autoimmune diseases. Therefore, patients with primary aldosteronism should be assessed for the presence of inflammatory skin diseases, including LLP.

\section{Consent}

The examination of the patient was conducted according to the principles of the Declaration of Helsinki.

The authors certify that they have obtained all appropriate patient consent forms, in which the patients gave their consent for images

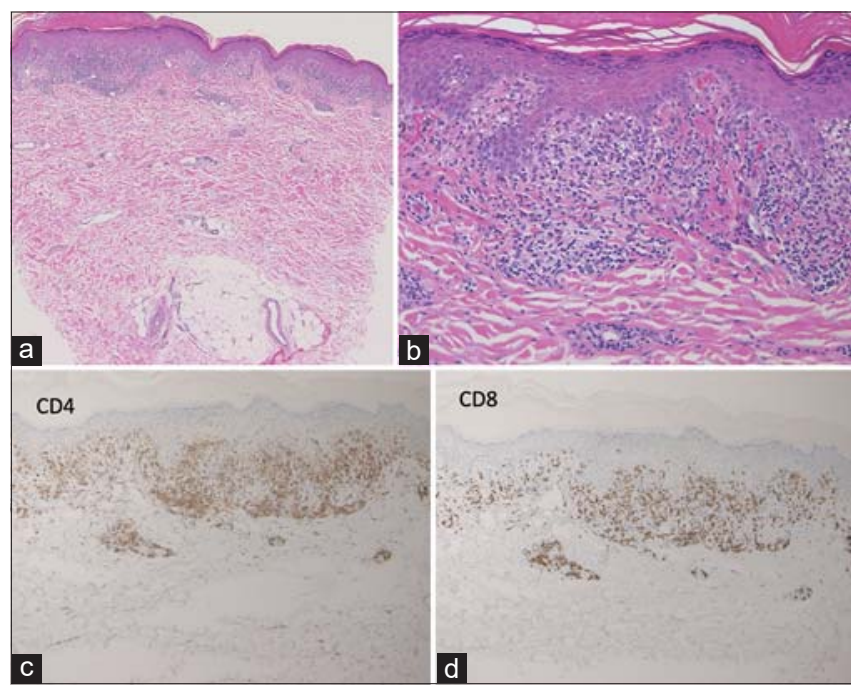

Figure 2: (a-b) A saw-tooth appearance of the epidermis and severe liquefaction degeneration (H\&E; (a) 200x); a band-like lymphocytic infiltration in the upper dermis. (c-d) Lichenoid infiltration into the dermis composed of CD4+ and CD8+ T lymphocytes; CD8+ T lymphocytes infiltrating into the epidermis (H\&E, 200x).

and other clinical information to be included in the journal. The patients understand that their names and initials will not be published and due effort will be made to conceal their identity, but that anonymity cannot be guaranteed

\section{REFERENCES}

1. Krysiak R. Okopien B. Coexistence of primary aldosteronism and Hashimoto's thyroiditis. Rheumatol Int. 2012;32:2561-3.

2. Kabbash C, Laude TA, Weinberg JM, Silverberg NB. Lichen planus in the lines of Blaschko. Pediatr Dermatol. 2002;19:541-5.

3. Gönül M, Atay S, Cemil BC, Akış HK, Gökçe A. A case of unilateral linear lichen planus: Related to orthopedic prosthesis or not? Postepy Dermatol Alergol. 2015;32:310-1.

4. Lai YC, Yew YW. Lichen planus and lichenoid drug eruption after vaccination. Cutis. 2017;100:E6-20.

5. Hadian Y, Chen YC, Eastham DV, Schulman JM, Sood A. Radiation induced lichen planus - an uncommon side effect. Dermatol Online J. 2019;25:13030.

6. Herrada AA, Contreras FJ, Marini NP, Amador CA, González PA, Cortés CM, et al. Aldosterone promotes autoimmune damage by enhancing Th17-mediated immunity. J Immunol. 2010;184:191-202.

7. Baykal Selcuk L, Sahin M, Arıca DA, Orem A, Karaca Ural Z, Yayll S. Impairment of myocardial functions and arterial stiffness in patients with lichen planus. An Bras Dermatol. 2020;95:180-6.

Copyright by Natsuko Matsumura, et al. This is an open access article distributed under the terms of the Creative Commons Attribution License, which permits unrestricted use, distribution, and reproduction in any medium, provided the original author and source are credited. Source of Support: Nil, Conflict of Interest: None declared. 